\title{
No evidence for involvement of IL-4R and CD11B from the IBD1 region and STAT6 in the IBD2 region in Crohn's disease
}

\author{
Dirk J de Jong ${ }^{\star}, 1$, Barbara Franke ${ }^{2}$, Anton HJ Naber ${ }^{1}$, Judith JHT Willemen ${ }^{2}$, Angelien JGAM
} Heister $^{2}$, Han G Brunner ${ }^{2}$, Carolien GF de Kovel ${ }^{2}$ and Frans A Hol ${ }^{2}$

\author{
${ }^{1}$ Department of Gastroenterology \& Hepatology, University Medical Center Nijmegen, The Netherlands; ${ }^{2}$ Department \\ of Human Genetics, University Medical Center Nijmegen, The Netherlands
}

Linkage studies have identified the inflammatory bowel disease (IBD)1 locus on chromosome 16 and the IBD2 locus on chromosome 12 to be involved in Crohn's disease. NOD2/CARD15 was identified as the gene of interest within the IBD1 region. However, linkage to this region could not be explained by NOD2/ CARD15 alone. Here we set out to assess the association of additional candidate genes from the IBD1 and IBD2 loci with Crohn's disease using transmission disequilibrium testing in patient-parent triads. No significant association was observed with genetic variants in the genes coding for interleukin-4 receptor gene (IL-4R), CD11B and signal transducer and activator of transcription type 6 (STAT6). Results for IL-4R were not affected by exclusion of all families carrying one of three risk alleles in NOD2. From this we conclude that IL-4R and CD11B in the IBD1 region and STAT6 in the IBD2 region are not involved in Crohn's disease in this Dutch cohort.

European Journal of Human Genetics (2003) 11, 884-887. doi:10.1038/sj.ejhg.5201058

Keywords: Crohn's disease; IBD; IL-4R; CD11B; STAT6; ITGAM

\section{Introduction}

Genetic susceptibility to inflammatory bowel disease (IBD) depends on the contribution of multiple genes, rather than a single genetic factor. ${ }^{1}$ Linkage studies revealed the IBD1 region on chromosome 16 to be involved in Crohn's disease (CD), and to a lesser extent in ulcerative colitis (UC) and the IBD2 region on chromosome 12 in UC, and to a lesser extent in $\mathrm{CD}$. Both regions harbor many genes, some of which encode proteins involved in immune response, which are good candidates for CD. Recently, the NOD2/ CARD15 gene was identified as a CD susceptibility gene within the IBD1 region with a population attributable risk

${ }^{*}$ Correspondence: Dr DJ de Jong, Department of Gastroenterology \& Hepatology, University Medical Center Nijmegen, PO Box 9101, $6500 \mathrm{HB}$ Nijmegen, The Netherlands. Tel: + 3124 3614760; Fax: + 3124 3540103; E-mail: d.dejong@mdl.umcn.nl

Received 24 September 2002; revised 7 April 2003; accepted 23 May 2003 of $25-30 \% .^{2}$ However, the NOD2/CARD15 gene cannot by itself explain the magnitude of the genetic linkage detected in previous studies. Hampe et $a l^{3}$ recently provided strong evidence for the existence of another IBD gene within IBD1. The interleukin-4 receptor $(I L-4 R)$ gene forms an excellent candidate due to its location, the gene coincides with a strong linkage peak in IBD1 (Figure 1), and its function in a signaling pathway leading to T-cell differentiation, which is important in inflammatory responses. ${ }^{4,5}$ Another putative candidate in IBD1 is the gene encoding complement receptor type 3 (CD11B). $\mathrm{CD} 11 \mathrm{~B}$ is a leukocyte surface antigen involved in granulocyte cell adhesion and is upregulated in patients with IBD. ${ }^{6}$ Within the IBD2 region, the gene encoding the signaltransducer-and-activator-of-transcription (STAT6) is located. STAT6 plays a role in the aforementioned IL-4R pathway, and therefore represents a good candidate for CD susceptibility. 5,7 The aim of the present study was to 


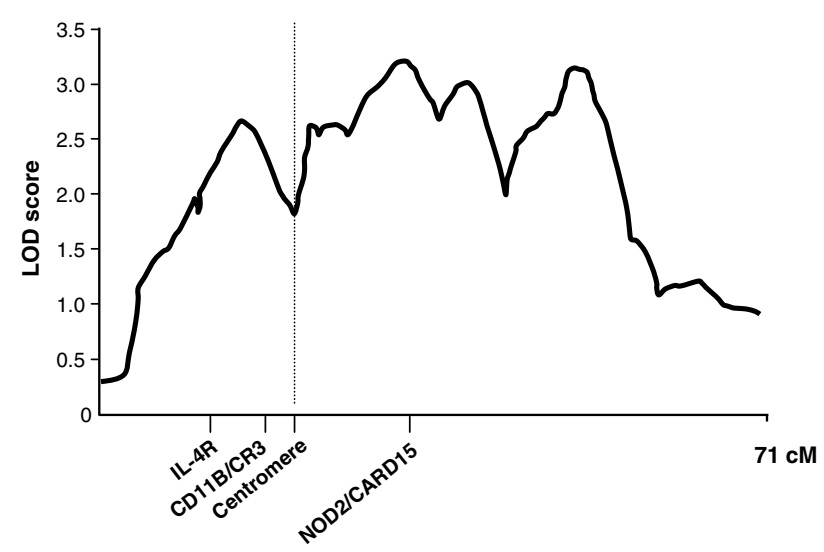

Figure 1 Location of the linkage peaks in the pericentromeric IBD1 region on chromosome 16 (16p12.316q13) obtained through different CD studies (modified from 3). The approximate locations of the genes coding for IL-4R, CD11B and NOD2/CARD15 are represented on the $X$-axis, the position of the centromere is indicated by a dashed line. cM: centiMorgan.

Table 1 Clinical characteristics of patients with CD

\begin{tabular}{lc}
\hline Characteristics & CD patients $(n=106)$ \\
\hline Gender (male/female) & $40 / 66$ \\
Age of onset (year) & $22.9 \pm 7.5$ \\
Location of disease & $32(30)$ \\
Ileum & $18(17)$ \\
Colon & $56(53)$ \\
Ileum and colon & $58(55)$ \\
History of bowel resection & $51(48)$ \\
History of fistula & \\
\hline
\end{tabular}

Results given as number of patients, percentage in brackets, or as mean $\pm S D$

determine whether genetic polymorphisms in candidate genes with immune regulatory functions located in IBD1 and IBD2 are associated with CD.

\section{Material and methods}

DNA was obtained from 110 Caucasian patients with confirmed CD, of which 106 unrelated patients (66 female) were included in the analysis, as were 147 of their parents (56 complete triads, 35 dyads, 15 patients without parents). All patients originated from The Netherlands. Clinical characteristics of the patients are shown in Table 1 . The transmission disequilibrium test (TDT)-PHASE package was used to test for association (www.hgmp.mrc.uk/ fdudbrid/software/unphased). This package allows inclusion of families with one or both parents missing. Applying Bonferroni's correction for multiple testing (number of tests $=4), P$-values $<0.0127$ were considered significant.

A fragment located in the $5^{\prime}$ region of the $C D 11 B$ gene was polymerase chain reaction (PCR)-amplified and singlestrand conformation polymorphism (SSCP)-analyzed to identify a suitable single nucleotide polymorphism (SNP) for association testing. The following primers were used: forward 5'-TGCTGGCATTACAGGCGTGA-3'; reverse 5'-GTAGGAGGCAGAAGTCTATC-3'. Aberrant bands were analyzed by direct sequence analysis on an ABI3700 automatic sequencer using BigDye terminator chemistry.

Several SNPs were typed using Pyrosequencing ${ }^{\circledR}$ technology, ${ }^{8}$ according to the protocol of the manufacturer. Primers for PCR amplification: IL-4R-I50V: forward primer 5'-biotin-CCAGATCTGTCCTCACATCCGT-3', reverse primer 5'-CATGCTCGCTGGGCTTGAAG-3'; IL4R-Q576R: forward primer 5'-biotin-CGCCGAAATGTCCTCCAGC-3', reverse primer 5'-CCCTGCTCCACCGCATGTA-3'; CD11B: forward primer 5'-biotin-TGCTGGCATTACAGGCGTGA-3', reverse primer 5'-GTAGGAGGCAGAAGTCTATC-3'; STAT6: forward primer $5^{\prime}$-AAGGGCTGAGATTCTTCGTGT- $3^{\prime}$, reverse primer $5^{\prime}$-biotin-CCCAAATTTGTGTTGTCACGTA-3'; NOD2-G908R: forward primer 5'-TACTGCAGAGGGAG GAGGACT-3', reverse primer 5'-biotin-CACCTGATCTCCC CAAGAAA. Sequence primers for pyrosequence analysis: IL-4R-I50V: 5'-CCGTTGTTCTCAGGG-3'; IL4R-Q576R: 5'CCGCATGTACAAACTCC-3'; CD11B: 5'-GCATATGAACAA GCTGAA-3'; STAT6: 5'-TGTACCTAGGATATGTTA-3'; NOD2-G908R: 5'-CCTTTTCAGATTCTGGG-3'.

The NOD2 C-insertion polymorphism was typed via PCR amplification of the genomic region and subsequent gel electrophoresis on a $6.6 \%$ polyacrylamide gel. ${ }^{9}$ PCR was conducted using the following primers: forward $5^{\prime}$ AATCCTTGAAGCTCACCATTG-3', reverse 5'-CAGGGGCC TTACCAGACT- $3^{\prime}$. Allelic bands were visualized by silver staining. The NOD2-R702S polymorphism was analyzed by restriction fragment length polymorphism analysis using HpaII. ${ }^{9}$ The primers used were: forward 5'-GAGCACTG GGGCCTGCTG-3' $\quad$ reverse $5^{\prime}$-GGATGGAGTGGAAGTG CTTG-3'.

\section{Results \\ CD11B and STAT6}

SSCP analysis of part of the $5^{\prime}$ end of the CD11B gene revealed a frequent polymorphism in the promoter region, identified as an $\mathrm{A} / \mathrm{G}$ variation at nucleotide position -324 (Genbank M76724; the first nucleotide 5' to the ATG start codon denoted -1). No transmission distortion of this particular $C D 11 B$ polymorphism was observed in the patients with CD $(P=0.548$; Table 2). For STAT6, we analyzed an A/G SNP (dbSNP identity: rs4559) in the 3' UTR, but here also no significant association of this polymorphism with $\mathrm{CD}$ was found $(P=0.190$; Table 2$)$.

\section{IL-4R}

For analysis of $I L-4 R$, we used two previously reported SNPs in the coding region of the gene ${ }^{10}$ (Table 2). Both SNPs concern $A / G$ variants leading to variation at the protein level (I50 V and Q576R). Although located in the same 
Table 2 Results of the TDT-phase analysis in 106 patients with CD

\begin{tabular}{|c|c|c|c|c|c|}
\hline Gene & SNP & Transmitted & Nontransmitted & OR & P-value \\
\hline IL-4R (I 50 V) & $\begin{array}{l}A \\
G\end{array}$ & $\begin{array}{r}120.2 \\
99.8\end{array}$ & $\begin{array}{r}121.4 \\
98.6\end{array}$ & $\begin{array}{l}1 \\
1.023\end{array}$ & 0.928 \\
\hline IL-4R (Q576R) & A & $\begin{array}{r}187.9 \\
32.1\end{array}$ & $\begin{array}{r}166.7 \\
53.3\end{array}$ & $\begin{array}{l}1 \\
0.533\end{array}$ & 0.028 \\
\hline IL-4R (Q576R)-NOD2 & $\begin{array}{l}A \\
G\end{array}$ & $\begin{array}{r}124.6 \\
23.4\end{array}$ & $\begin{array}{r}113.2 \\
34.8\end{array}$ & $\begin{array}{l}1 \\
0.609\end{array}$ & 0.157 \\
\hline CD11B & $\begin{array}{l}A \\
G\end{array}$ & $\begin{array}{r}143.3 \\
72.7\end{array}$ & $\begin{array}{r}150 \\
66\end{array}$ & $\begin{array}{l}1 \\
1.154\end{array}$ & 0.548 \\
\hline STAT6 & A & $\begin{array}{r}147.0 \\
73.0\end{array}$ & $\begin{array}{r}131.7 \\
88.3\end{array}$ & $\begin{array}{l}1 \\
0.740\end{array}$ & 0.190 \\
\hline
\end{tabular}

gene, the polymorphisms were in complete equilibrium with each other $(P=0.706)$. No association of the I50 V variant and $C D$ was found $(P=0.928)$. Analysis of $Q 576 \mathrm{R}$ revealed an OR of 1.86 for $576 \mathrm{Q}$, and a $P$-value of 0.028 . Although suggestive for association, this $P$-value could not be considered significant after Bonferroni's correction. To ensure that these latter findings were not obscured by mutations in NOD2/CARD15 in the same region, we typed the three major susceptibility mutations found in this gene (see below) and repeated the IL-4R Q576R analysis excluding patients carrying one or more NOD2/CARD15 variants $(n=36)$. This resulted in a nonsignificant $P$-value of 0.157 .

\section{NOD2/CARD15}

Three functional variants in NOD2 (R702W, G908R, 3020insC) were analyzed. ${ }^{9}$ Allele frequency of 3020insC variant in patients was $8.1 \%(n=105)$ with nine heterozygous and four homozygous subjects. The R702W variation was observed with a frequency of $13.2 \%$ in patients $(n=106), 26$ being heterozygous and a single one homozygous. Variant G908R was not observed. Four out of 105 patients were compound heterozygotes. The proportion of patients with at least one variant NOD2/CARD15 allele was $34.0 \%$. Recently, it has been shown that NOD2/CARD15 mutations are specifically involved in ileum-specific CD. ${ }^{11}$ When confining our analysis to patients with ileumspecific disease $(n=32), 46.9 \%$ possessed at least one variant NOD2/CARD15 allele.

\section{Discussion}

Recent findings suggest that approximately $25-30 \%$ of the genetic susceptibility in CD can be explained by mutations in NOD2/CARD15, a gene located in the IBD1 region. ${ }^{2}$ The broad-IBD1 region on chromosome 16 is extending from $16 \mathrm{p} 12.3$ to $16 \mathrm{q} 13$. At the boundary of this region at $16 \mathrm{p} 12.1$, the $I L-4 R$ gene is located. Although this gene can be regarded as a candidate for $\mathrm{CD}$, we found no significant association with two SNPs, I50 V and Q576R, located in the coding region of the gene. Both SNPs were in complete equilibrium with each other, which is in accordance with previous results of Olavesen et al. ${ }^{10}$ We re-evaluated the association with the Q576R variant after excluding families for which the patient carried at least one variant NOD2/ CARD15 allele. For this purpose, the three most prominent variants in NOD2/CARD15 were analyzed. At least one risk allele was found in $34 \%$ of all CD patients and in nearly $47 \%$ of patients with ileum-specific CD. Exclusion of patients with an NOD2/CARD15 risk allele did not result in a stronger association of the IL4R-Q576R allele with the disease, suggesting that the $I L-4 R$ gene is not a major risk factor for $C D$. The same holds for another candidate gene in IBD1 on $16 \mathrm{p} 11.2$, the $C D 11 B$ gene. In IBD2 on chromosome 12, no evidence was found for an association of the STAT6 gene with CD.

In conclusion, in this Dutch cohort no evidence for association was observed between $\mathrm{CD}$ and alleles of the genes encoding the IL- 4 receptor, CD11B or the STAT6 by TDT. However, the number of SNPs and patients analyzed in the present study is limited. Typing of additional SNPs in larger panels of patients will be needed to define putative risk haplotypes at each locus in more detail.

\section{Acknowledgements}

We thank our colleagues $M$ Schijvenaars and $S$ Hanssen for their technical assistance and $H$ Straatman for support with statistical analysis of the data. This study was supported by a grant of the Netherlands Digestive Diseases Foundation.

\section{References}

1 Satsangi J, Jewell DP, Bell JI: The genetics of inflammatory bowel disease. Gut 1997; 40: 572-574.

2 Cuthbert AP, Fisher SA, Mirza MM et al: The contribution of NOD2 gene mutations to the risk and site of disease in inflammatory bowel disease. Gastroenterology 2002; 122: 867-874.

3 Hampe J, Frenzel H, Mirza MM et al: Evidence for a NOD2independent susceptibility locus for inflammatory bowel disease on chromosome 16p. Proc Natl Acad Sci USA 2002; 99: 321-326.

4 Duncan DD, Swain SL: Role of antigen-presenting cells in the polarized development of helper $\mathrm{T}$ cell subsets: evidence for differential cytokine production by Th0 cells in response to antigen presentation by B cells and macrophages. Eur J Immunol 1994; 24: 2506-2514.

5 Dent AL, Hu-Li J, Paul WE, Staudt LM: T helper type 2 inflammatory disease in the absence of interleukin 4 and transcription factor STAT6. Proc Natl Acad Sci USA 1998; 95: $13823-13828$. 
6 Rogler G, Andus T, Aschenbrenner E et al: Alterations of the phenotype of colonic macrophages in inflammatory bowel disease. Eur J Gastroenterol Hepatol 1997; 9: 893-899.

7 Zhang S, Lukacs NW, Lawless VA, Kunkel SL, Kaplan MH: Cutting edge: differential expression of chemokines in Th1 and Th2 cells is dependent on Stat6 but not Stat4. J Immunol 2000; 165: $10-14$.

8 Fakhrai-Rad H, Pourmand N, Ronaghi M: Pyrosequencing: an accurate detection platform for single nucleotide polymorphisms. Hum Mutat 2002; 19: 479-485.
9 Hugot JP, Chamaillard M, Zouali $\mathrm{H}$ et al: Association of NOD2 leucine-rich repeat variants with susceptibility to Crohn's disease. Nature 2001; 411: 599-603.

10 Olavesen MG, Hampe J, Mirza MM et al: Analysis of singlenucleotide polymorphisms in the interleukin-4 receptor gene for association with inflammatory bowel disease. Immunogenetics 2000; 51: 1-7.

11 Ahmad T, Armuzzi A, Bunce M et al: The molecular classification of the clinical manifestations of Crohn's disease. Gastroenterology 2002; 122: 854-866. 